Full citation:

M. Vadym, "Exploration of Scenario-based Simulations for Stress Benchmarking in Swiss Public Service," 2020 7th Swiss Conference on Data Science (SDS), Luzern, Switzerland, 2020, pp. 23-28, doi: 10.1109/SDS49233.2020.00012.

doi: 10.1109/SDS49233.2020.00012

Link to the final, published article in IEEE Xplore:

https://ieeexplore.ieee.org/document/9145028

Copyright (C) 2020 IEEE. by The Institute of Electrical and Electronics Engineers, Inc. All rights reserved. 


\title{
Exploration of Scenario-based Simulations for Stress Benchmarking in Swiss Public Service
}

\author{
Mozgovoy Vadym \\ Swiss Graduate School of Public Administration (IDHEAP) \\ University of Lausanne \\ Lausanne, Switzerland \\ vadym.mozgovoy@unil.ch
}

\begin{abstract}
Statistical interpretation of stress-related indicators collected through wearable biosensors often relies on benchmarking, especially in the context of stress management interventions. However, it remains unclear how to construct stress level benchmarks for group stress-related indicators using limited historical data. This study examines whether the method of numerical simulation of stress-related responses could contribute to constructing benchmark curves. Experimental data consists of physiological and non-physiological signals of 18 Swiss public servants collected through wearable biosensors. This study draws upon Stress Pattern Recognition algorithm and Markov Chain modeling for simulating emotional responses according to specified data-driven scenarios of high and low stress. Proposed method allows constructing benchmark curves for an Overarousal Index. Results demonstrate that numerical simulation based on small datasets can be used effectively for constructing stress level benchmarks. The findings contribute to methodological knowledge in statistical learning on Stress Pattern Recognition algorithms and Markov Chains modeling by expanding their application to a new field of emotional response simulation according to scenarios.
\end{abstract}

Keywords - Numerical simulation, Markov chains, bootstrap, benchmark, biosensor, wearables, stress, electronic stress management, organization, Swiss public administration

\section{INTRODUCTION}

Supporting psychological well-being and decreasing stress in the workplace is important for achieving optimal productivity. Stress could be particularly detrimental to performance, and organizations are increasingly use shortterm stress management interventions for averting its negative consequences and well-being promotion. Timely identification of stress as well as benchmarking, or comparison between normal and actual stress state, are necessary to support decision-making on stress management.

Physiological and non-physiological signals can serve as foundation for remote algorithm-based stress pattern recognition. However, there is a methodological gap in the literature concerning construction of benchmark curves in the presence of limited datasets. Random process-based numerical simulation might contribute to constructing benchmark curves for stress monitoring, when the historical data is limited. It widely draws upon Monte-Carlo methods. They represent an integral part of Markov Chains Monte Carlo

Acknowledgment: This research has been supported by the Swiss National Science Foundation (SNSF) grant no. 172740 methods (MCMC) and dynamic simulation modeling methods comprising system dynamics, discrete-event simulation and agent-based modeling [1]. MCMC serves to approximate distribution of parameters of interest by random sampling from a target probability density function [2]. These methods have applications across different domains. For instance, MCMC is widely used in computer-based simulation of experiments in physics in order to understand phase transition and other physical behaviors [2]. Theoretical chemistry simulates chemical reactions through MCMC[3]. MCMC method is also used in DNA sequence simulation and analysis [4]. Other application domains of MCMC include finance [5], meteorology [6], biology [7], and engineering [8].

Current study addresses the literature gap on benchmarks for stress pattern recognition with limited historical data by suggesting MCMC simulation for the construction of stressrelated benchmark curves. Accordingly, this study addresses the following research question: could numerical simulation through MCMC and bootstrap methods contribute to stress benchmarking in the workplace? This research is conducted in the context of Swiss public administration where psychological well-being of public servants is a core public value. Interestingly, highly motivated public employees are likely to suffer from stress more that those without motivation, according to the research conducted in Swiss public service [5]. The aim of this research is to explore whether numerical simulation can be used to develop stress-related benchmarks with small datasets in the workplace, using the data on fiscal office workers from Switzerland,

\section{REMOTE STRESS IDENTIFICATION}

Stress is defined as a relationship between an individual and an environment appraised as taxing or exceeding resources under control, thus threatening personal well-being" [6]. According to this definition, the concept of arousal is critical for stress identification. Arousal level represents a capacity that an individual disposes to perform tasks [7]. It can vary according to low, mid, and high level. According to the Yerkes-Dodson law explaining the relationship between arousal and performance [7], very low arousal levels can lead to inertia, boredom, dissatisfaction, and reduced performance. Mid-level arousal supports optimal performance, extends the length of progress, prepares for change, and boosts creativity. Arousal can exceed mid-level, when individual performs cognitive or physical tasks. When arousal reaches very high level or stay at high level for a prolonged period of time, it refers to the state of Overarousal. An overaroused individual 
is highly likely to experience stress, profound and long-lasting decline of performance.

Stress pattern recognition involves supervised or unsupervised machine learning approaches [8,9] Selection between these two approaches depends on the study context [10]. Previous research has confirmed that machine learning algorithms can identify stress or stress-relates states. Statistical learning studies on SVM-kNN [11], LDA [12], Convolutional Neural Networks [13], Logistic regression [14] and other algorithms show high predictive accuracy of stressrelated classification, frequently exceeding 70\% [10]. Controlled laboratory conditions provide higher level of accuracy, compared to the workplace or daily life setting.

In the workplace setting, sensorial signals allowing to identify stress-related patterns are collected during brief time periods of remote stress monitoring. Remote stress identification using algorithms is based on sensorial physiological and non-physiological signals. Emitted signals reflect transitions between various emotional states that individuals may have over time. Most commonly used physiological signals include Galvanic Skin Response (GSR), Heart Rate (HR), Respiratory Rate (RR). Typically, such data is collected in organizations during short interventions.

Construction of stress-related benchmarks based on sensorial signals is one of morbidity (dysfunction) tracking practices, which raises challenges from statistical methodology perspective. First, stress patterns would not be "typical" or the same across different work environments. Stress levels may vary across activity sectors, occupations, and job positions. For example, normally experienced stress level would differ for fiscal office workers and police officers, and manager are likely to experience higher stress than line workers. Second, benchmarks could be time-dependent. Third, they may exhibit seasonality influence, because a job could commonly require to work intensively at some periods, but not at others. Thus, benchmark curves may change over time. Fourth, they may also incorporate stochastic variance. For addressing these and similar methodological issues, morbidity tracking practices frequently rely on large temporal series spanning over months, years or even decades [15, 16].

While morbidity tracking requires large time series, workplace stress monitoring typically consists of short time series based on data collected during context-specific workplace stress monitoring. In some cases, time horizon of sensorial data collected through wearable devices spans only over several months. There is a gap in statistical methodological literature regarding construction of stressrelated benchmark curves with limited historical data, and our study aims to address this research problem.

\section{RESEARCH METHODS}

Present study on remote stress pattern recognition aims to expand methodological knowledge on data pre-processing under conditions of limited longitudinal data availability [17]. Current research follows several steps. They include (1) data labeling with Gaussian Mixture Model (GMM); (2) datadriven scenario formulation with block bootstrap; and (3) numerical simulation with Markov Chain Model.

First, we use GMM for labeling stress-related features according to several classes: Relaxation (R), Arousal (A) and Overarousal $(\mathrm{O})$. The resulting data presents sequential realizations of those 3 classes. For example, 'RRRAAAO' represent sequential chain realization where $\mathrm{R}$ transits to $\mathrm{A}$ at time 4 and transits to $\mathrm{O}$ at time 7. Methodological procedures for GMM are well established in the literature [18, 19].

Second, we make a block resampling of consecutive emotional episodes from labeled dataset. We define resampling units as class $i$ realizations before transiting to another class $j$, according to the original data structure. For example, dataset "RRRRAAOO" has three resampling units: "RRR" is a first unit, "AAA" is a second unit and "OO" is a third unit. Then, for each sample, we count the amount of transitions, i.e. transition frequencies, from one class $\left(c_{i}\right)$ to another class $\left(c_{j}\right)$ that we denote as path: $c_{i j}$. We also count number of realizations excluding transitions from one class to another. It corresponds to realizations when chain remains in the same class that we denote as $c_{i i}$. Accordingly, addition of paths $c_{i j}$ and $c_{i i}, \forall i, j \in\{R, A, O\}$ yields a total number of realizations in data sample. We obtain distributions of each path through a resampling procedure. Bootstrap distributions of the biggest path $c_{R R}$ will further serve to develop scenarios called "High Stress" and "Low Stress".

Third, we perform Markov Chain simulation of benchmark curves under scenarios of high and low stress. Next, we display them against "Overarousal" index. Therefore, we introduce a block bootstrap method and Markov Chain model further in this section.

\section{A. Block Bootstrap}

Bootstrap refers to a general resampling procedure for estimating sampling distributions which is approximate and conditional on the observed data [20]. The algorithm corresponds to drawing a large number of bootstrap samples, $y^{*}(1), y^{*}(2), \ldots, y^{*}(B)$. We evaluate a statistic of interest for each sample $\hat{\theta}^{*}(b)=\hat{\theta}\left(y^{*}(b)\right)$ for $b=1,2, \ldots, B$, which compose a distribution [21]. We estimate the sampling distribution for general stationary observations, using a block bootstrap [3, 22]. Observations well separated in time are assumed to be nearly uncorrelated, and time series of length $n$ can be factored as $n=b \cdot l$, where $b$ and $l$ are integer, and $l$ should be large enough to retain correlation structure. We apply block bootstrap resampling to sequential data with inhomogeneity regions in Stationary Categorical Time Series [23]. We model a sequence of stress-related conditions as a Markov process with irreducible transition matrix, corresponding to a stationary process.

Also, the sequence contains inhomogeneity regions. For example, exploratory data analysis shows that work period in the beginning of November is calmer than end of Novembermid of December. The latter period may correspond to high work intensity in fiscal office when it is time to finalize annual accounting reports. For this reason, we use a block bootstrap with at least two blocks in order to estimate the distribution of transition frequencies between stress-related states. Thus, we define four equally spaced regions for random sampling with replacement consisting in 100 samples per block and accounting for $80 \%$ of observations at each sampling: $b=4$, $l=3533, n=b \cdot l=14^{\prime} 132$.

\section{B. Markov Chain Model}

Markov chain is a model generating sequential output, characterized by a set of states $Q$, emitting symbols and a set of transitions between states [19]. Each transition between states has corresponding transition probability $p_{i j}$. It represents a conditional probability of going to state $j$ in the 
next step from state $i$ in current step. Sum of transition probabilities at state $\mathrm{j}$ equals to $1, \sum_{j} p_{i j}=1$, for all $\mathrm{j} \in Q$ $[19,24]$. First-order Markov process is a random process $\{X(t), t \in T\}, t_{o}<t_{1}<\cdots<t_{n}$, if in any time $t$ conditional CDF of $X\left(t_{n}\right)$ depends only on $X\left(t_{n-1}\right)$ given values of $X\left(t_{0}\right), X\left(t_{1}\right), \ldots, X\left(t_{t-1}\right)[20,24]$, presented as follows [24]:

$$
\begin{aligned}
& P\left[X\left(t_{n}\right) \leq x_{n} \mid X\left(t_{n-1}\right) \leq x_{n-1}, \ldots, X\left(t_{0}\right) \leq x_{0}\right]= \\
& P\left[X\left(t_{n}\right) \leq x_{n} \mid X\left(t_{n-1}\right) \leq x_{n-1}\right]
\end{aligned}
$$

A distribution $p^{*}(x)$ corresponding to a random process $\{X(t), t \in T\}$ is stationary, when each step in the chain leaves this distribution invariant [18]. When all states in chain communicate, this chain is irreducible [24]. If time Markov chain is finite, irreducible and discrete it has a unique stationary distribution [25]. In order to estimate transition probabilities, we estimate the following ratio [19]:

$$
p_{i j}=\frac{c_{i j}}{\sum_{k} c_{i k}}
$$

where $c_{i j}$ refers to the number of times that class $j$ follows class $i$, representing an absolute frequency. Further, $c_{i j}$ is normalized by the sum of transitions $i$ over $k$, which is $\sum_{k} c_{i k}$.

We model our data as a sequential realization of classes, labeled as follows: Relaxation (R), Arousal (A), and Overarousal (O) observed over time $t$. Correspondingly, $i, j \in$ $\{R, A, O\}, k=3$. Transition probabilities from one state to another can be estimated with $p_{i j}$ coefficient, where $c_{i j}$ will correspond to absolute frequency of passing from condition $i$ to condition $j$. Normalization constant corresponds to summing absolute frequencies over all possible states.

We retain first-order Markov Chain process, because principle feature of emotional arousal used in this study, Galvanic Skin Response (GSR), exhibit strong and positive auto-correlation (AR) pattern with highly pronounced firstodder component according to the output of AR(15) model.

\section{STUDY PARTICIPANTS}

Sensorial data was collected during study in Municipal Fiscal Administration Office in Switzerland. Experimental timeline was determined in coordination with the Office management. Research team distributed consent forms among potential participants. Individuals that gave formal consent were recruited. Sample of 18 participants includes 6 males and 12 males working across 7 different departments. The biggest departments were "Construction Management", "City Chancellery" and "Tax Office", where the latter comprised 7 participants. Participants wore biosensor devices in the workplace for approximately 2 months from 1.10 .2018 to 18.12.2018. It involved periods of low and high work intensity in order to ensure sufficient variability in stress-related physiological response. According to the research assumptions, first half of November may be calmer compared to the end of November - mid of December, when the burden of finalizing accountability increases in Tax Office.

\section{DATA AND MEASUREMENT}

This study uses experimental observations from wearable devices, also called wearables. Biosensors integrated in wearables continuously collect data on physiological signals of research participants. Each extracted feature consists of 60 seconds of averaged GSR and Heart Rate (HR) and Motion Activity (MA) without overlap.

\section{NUMERICAL SIMULATION PROCEDURES}

\section{A. Data labeling with GMM}

We fit a 3-Dimensional Gaussian Mixture Model (GMM) with standardized GSR, HR and MA features (Fig. 1). We identify clusters corresponding to the state of R, A and $\mathrm{O}$. Next, we label input features accordingly, in line with previous research [17].

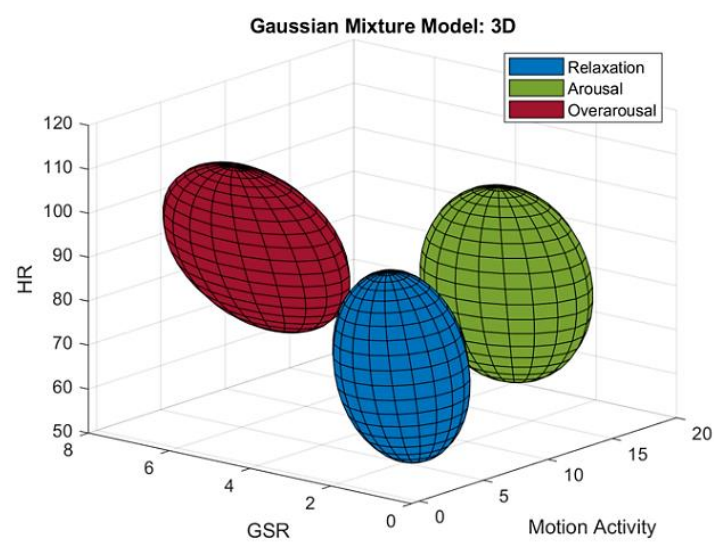

Fig. 1. Gaussian Mixture Model with 3 clusters in 3D

\section{B. Data-Driven Scenario Formulation}

Stationary categorical series contain inhomogeneity regions, described earlier. Consequently, we apply a block bootstrap for estimating the distribution of unnormalized transition frequencies $c_{i j}$, i.e. transition counts in each sample. Figure 2 shows a mixture distribution of path $c_{R R}$. In line with previous classification analysis [17], $\mathrm{R}$ is a most frequent state of individuals. Changes in the density of $\mathrm{R}$ episodes correspond to the changes in emotional states of users. Some device users remain in phase $\mathrm{R}$ for longer time in periods of lower stress and work intensity; accordingly, they transit from state $\mathrm{R}$ to state $\mathrm{O}$ and $\mathrm{A}$ less frequently. Other device users remain in phase $\mathrm{R}$ for shorter time in periods of high higher stress and work intensity; as a result, they transit from $\mathrm{R}$ to $\mathrm{O}$ and A more frequently. Therefore, observations grouped around 0.25 and 0.75 quantile lines, may correspond to "low stress" and "high stress" transition frequencies ( Fig. 2).

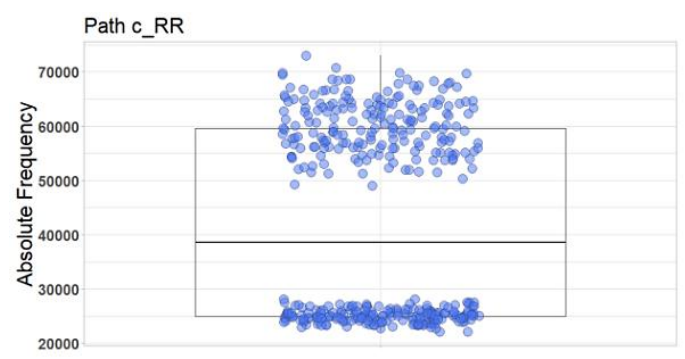

Fig. 2. Distribution of transition frequncies $c_{R R}$ with jittering

We develop low stress and high stress scenarios (Table 1). We assume that under High Stress Scenario users spend less time in state $\mathrm{R}$, then transition frequencies to state $\mathrm{R}$ from all other states are reduced. In parallel, users transit more regularly to state O from other states. In Low Stress Scenario, users spend more time in state R. So, transition frequencies to state $\mathrm{R}$ from all other states are increased. At the same time, users transit less regularly to state $\mathrm{O}$ from other states. Low frequency values of $\mathrm{R}$ episodes may be concentrated around 0.25 quantile line, and high frequency values of $\mathrm{R}$ episodes may be concentrated around 0.75 quantile line (Fig. 2). Therefore, we assume that 0.25 quantile marks the decreased 
path frequency, 0.75 quantile marks the increased path frequency while mean of bootstrap distribution reflects typical transition frequency.

TABLE I. SCENARIO FORMULATION

\begin{tabular}{|c|c|c|}
\hline \multirow{2}{*}{ Path Frequency } & \multicolumn{2}{|c|}{ Stress Pattern Scenarios } \\
\cline { 2 - 3 } & High Stress & Low Stress \\
\hline$c_{R R}$ & $\mathrm{q} 0.25$ & $\mathrm{q} 0.75$ \\
\hline$c_{R A}$ & mean & mean \\
\hline$c_{R O}$ & $\mathrm{q} 0.75$ & $\mathrm{q} 0.25$ \\
\hline$c_{A R}$ & $\mathrm{q} 0.25$ & $\mathrm{q} 0.75$ \\
\hline$c_{A A}$ & mean & mean \\
\hline$c_{A O}$ & mean & mean \\
\hline$c_{O R}$ & $\mathrm{q} 0.25$ & $\mathrm{q} 0.75$ \\
\hline$c_{O A}$ & mean & mean \\
\hline$c_{O O}$ & $\mathrm{q} 0.75$ & $\mathrm{q} 0.25$ \\
\hline
\end{tabular}

Next, we estimate lower quartile, upper quartile and mean of absolute path frequencies, $c_{i j}$. Then, we compute $p_{i j}$ coefficient by weighting absolute frequencies according to their contribution to the class so that for any class $i$, $\sum_{j=1}^{N} p_{i j}=1$. Normalized frequencies corresponding to "Low Stress" scenario and "High Stress" Scenario are shown in Figures 3 and 4. Finally, we run stochastic simulations of stress-related states under distributional assumptions of High Stress and Low Stress Scenarios.

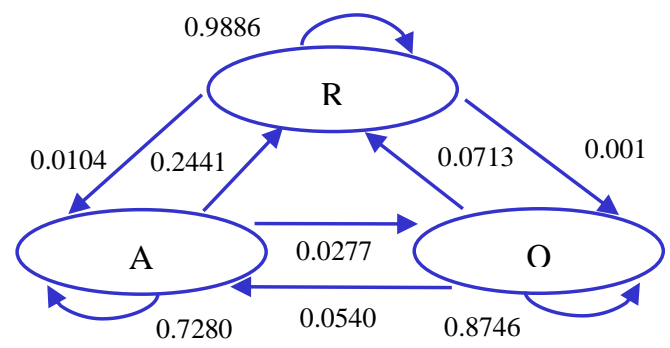

Fig. 3. State Diagram for Low Stress Scenario

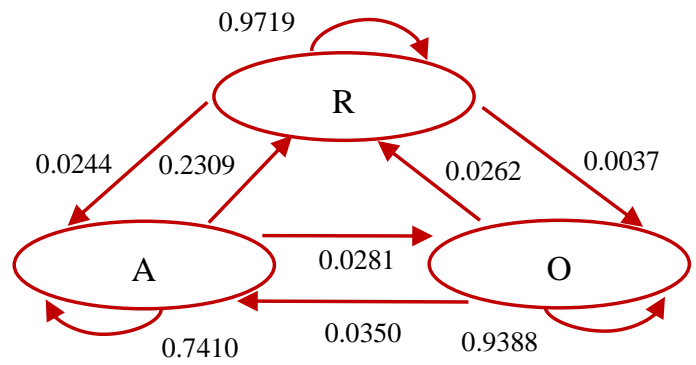

Fig. 4. State Diagram for High Stress Scenario

\section{Numerical Simulation with Markov Chains}

We simulate benchmarks for "Overarosal" Index based on Markov Chain models under scenarios of Low Stress and High Stress. We consider 20 pseudo-users wearing devices over 31 days. We assume that transitions between states happen every minute and devices are active 8 hours per day. We generate 480 daily realizations, i.e. $60 \mathrm{~min} \times 8$ hours $=$ 480 realizations/day. Overall length of generated sequence is 14880 , i.e. 480 transitions/day $\times 31$ days $=14$ ' 880 transitions/month. Original features consist in nonoverlapping sliding-window average of 60 seconds (i.e. one per minute). In line with the original dataset characteristics, Markov Chain generates realization each minute deciding whether to transit to different state or remain at current state at every step.

Next, we compute daily sum of "Overarousal" episodes for each pseudo-user, average it out, and construct simulated index of "Overarousal" (Fig. 5). The Red smoothed line shows collective "Overarousal" realizations under High Stress model scenario. Blue smoothed curve shows collective "Overarousal" realizations under Low Stress model scenario. Those curves may represent upper and lower thresholds in stress monitoring. Points around red and blue exponentially smoothed lines represent averaged daily outcomes per scenario. Black curve is a pseudo-index of "Overarousal" constructed by block resampling from original data (Fig. 5), considering 480 daily transitions for 31 days, and repeating the resampling procedure 20 times for 20 pseudo-users.

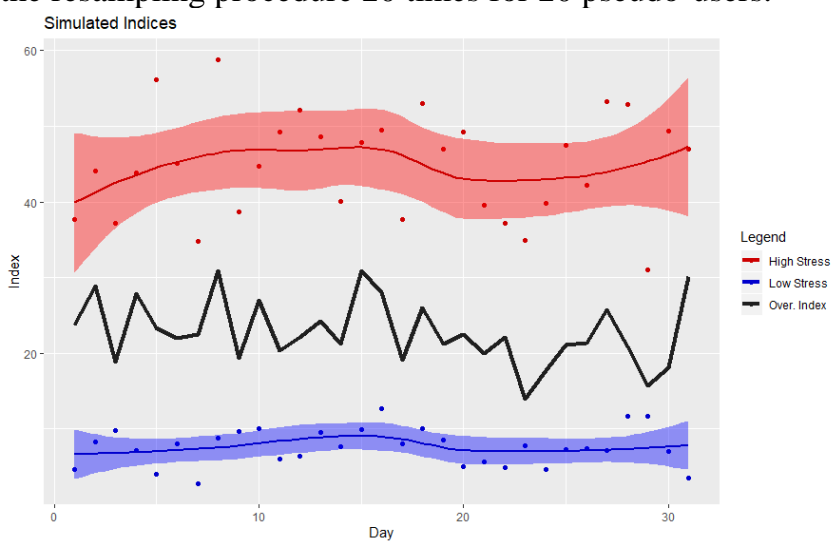

Fig. 5. Stress-Related Overarousal Index with Benchmarks

Results show that stress-related Overarousal index constructed via GMM classifier can be developed, using limited historical data. Benchmark curves establishing limits of high and low stress can be used in addition to Overarousal index. Thus, analytical approach combining Stress-Related Overarousal Index and benchmark curves facilitates interpretation of sensorial physiological signals.

\section{DISCUSSION}

\section{A. Findings}

Present study shows that stress-related benchmarks can be constructed with limited historical data through the use of classification algorithm, block bootstrap, and Markov Chain modeling. Our study extends the application of classification algorithms for constructing stress-related indices to a new field of emotional response simulation scenarios at group level in a broad workplace context. For achieving this objective, we use a block bootstrap for data-driven scenario formulation, and Markov Chain models for simulating benchmark curves under specific assumptions.

More specifically, our study contributes to public management literature by making a methodological advance allowing to examine stress-related indicators. We find that stress-related benchmarks with limited historical data may be effectively used in Swiss public administration. Introduction of wearable biosensor technologies in Public Sector may contribute to digital transformation of the workplace [26] and strengthen Digital Era Governance Model of public management [27]. 


\section{B. Methodological Implications}

Present study shows that numerical simulation allows identifying stress-related indicators in conditions when only a small data set is available in the workplace. Furthermore, our findings show that stress patterns can be recognized at group level and interpreted accordingly. From methodological perspective, these findings imply that both systematic and a non-systematic use of biosensor technologies could support stress benchmarking in a broad range of organizations as social groups.

Bootstrap methods and numerical simulation may facilitate the use of relatively small data corresponding to nonsystematic or restricted exploitation of wearables. Bootstrap methods allow to learn distributional characteristics from relatively small datasets and integrate them into simulation models. Further, block resampling schemes could increase a presence of observations corresponding to certain regions in the dataset.

Our findings imply that combined use of Overarousal index and benchmark curves enhances the interpretation of stress-related indicator with limited historical datasets. Estimation of transition probabilities may be coupled with qualitative analysis or questionnaire that do not stem from sensor data. Questionnaires can help to refine the identification of seasonality or inhomogeneity periods of stress in the workplace. Those periods may occur during shifts in the work complexity, work intensity or other factors. Qualitative assessment from employees may facilitate distinguishing between inhomogeneity regions, confirm data labeling, and justify benchmarks.

\section{Practical Implications}

From practical standpoint, stress-related indicators combining Overarousal index with benchmark curves may facilitate timely identification of excessive stress in the workplace. It may support managerial decision-making on stress management through isolated psycho-social interventions or comprehensive stress-management programs. Timely stress identification at group level may also support managerial decision-making on stress prevention through job design, workload optimization, and other human resource management practices. Finally, they may contribute to development of new occupational health and safety policies. These practical steps may result in decreasing stress as well as increasing productivity and psychological well-being in public service in Switzerland, in other countries and organization types.

Group-level use of sensory data for stress recognition would be an effective practical tool to enhancing psychological well-being in the workplace. Wearable technologies may provide new opportunities for electronic stress management, especially in the light of ongoing Public sector digitalization. Yet, the use of wearable technologies in the workplace must be authorized, ethical, and legal. Collective-level indicators may contribute to protection of information privacy and non-identifiability of individual employees. Recording of sensorial information in databases may be done in blinded way and activated only when certain threshold of users is met.

Wearables may be more expensive stress measurement tools than surveys. However, they bring additional opportunities for organizations. The range of opportunities depends on the built-in functionalities and relationships with vendors that may support device exploitation with additional data-intensive products and services. Relevant choice of resource access strategy for wearables may optimize their cost. Yet, organizations may choose between outsourcing, strategic alliances or internal development of data-intensive products which also influence cost of maintaining wearables in organizations [28]. Outsourcing relationships can sometimes lead to costly customization services. For example public administrations more frequently choose internal development for procuring information technologies for human resource management [28]. In this case, internal data management and development of data-intensive services can cut costs, making data and privacy protection more reliable, compared to outsourcing or de-centralized development.

\section{Limitations}

In spite of contribution to the literature, present study is subject to three main limitations. First, the GMM approach does not identify stress. However, the goal of present research is rather to identify classes with low, medium and high arousal associated with stress, and this goal is achieved.

Second, this model is oriented towards identification of common stress-related patterns at group level. It does not reflect individual heterogeneity in physiological stress-related responses. Therefore, Overarousal index and stress-related benchmarks based on this classifier would rather reflect general and shared trends in stress-related response at group level, mitigating individual particularities.

Third, obtained benchmarks are model-dependent. Stressrelated pattern of Swiss fiscal administration impacts transition frequencies through GMM. Stress-related benchmarks may vary substantially across different workplaces, and should be used with caution.

\section{E. Future Research}

Research should examine the validity of our methodological approach. Future studies should consider alternative classifiers for stress pattern recognition. They may explore construction of benchmark curves incorporating seasonality patterns and alternative simulation models. Future research may address the validity of auxiliary artifacts for stress-management, such as stress pattern recognition models, group-level indicators of stress and benchmarks curves. Future research may elaborate mixed methodologies, relying on triangulated biosensor data, numerical simulation methods, and stress-related benchmarking.

\section{CONCLUSION}

Our study examines a methodology for constructing stress-related benchmarks for Overarousal index, and it shows that numerical simulation is effective for identifying workplace stress with small dataset avaliability. This work contributes to emergent research on simulation of stressrelated responses according to specified scenarios from statistical methodology perspective. While these the benchmarking result are obtained in the context of Swiss public administration, empirical analysis confirms that numerical simulation may be effectively used across a broad range of organizations. We should build on this opportunity to improve timely stress identification, develop prevention of its negative consequences, and support enhancement of psychological well-being in the workplace over time. 


\section{REFERENCES}

[1] D. A. Marshall et al., "Selecting a dynamic simulation modeling method for health care delivery researchPart 2: Report of the ISPOR Dynamic Simulation Modeling Emerging Good Practices Task Force," Value in health, vol. 18, no. 2, pp. 147-160, 2015.

[2] W. S. Kendall, F. Liang, and J. S. Wang, Markov Chain Monte Carlo: Innovations and Applications: World Scientific, 2005.

[3] M. R. Chernick and R. A. LaBudde, An Introduction to Bootstrap Methods with Applications to R: Wiley, 2014.

[4] G. K. Chen, P. Marjoram, and J. D. Wall, "Fast and flexible simulation of DNA sequence data," Genome research, vol. 19, no. 1, pp. 136-142, 2009.

[5] D. Giauque, S. Anderfuhren-Biget, and F. Varone, “ Stress perception in public organisations: Expanding the job demands - job resources model by including public service motivation," Review of Public Personnel Administration, vol. 33, no. 1, pp. 58-83, 2013.

[6] R. S. Lazarus, Emotion and adaptation: Oxford University Press on Demand, 1991.

[7] R. M. Yerkes and J. D. Dodson, "The relation of strength of stimulus to rapidity of habit-formation," Journal of comparative neurology and psychology, vol. 18, no. 5, pp. 459-482, 1908.

[8] A. de Santos Sierra, C. Sanchez Avila, J. Guerra Casanova, and G. Bailador del Pozo, "A StressDetection System Based on Physiological Signals and Fuzzy Logic," IEEE Transactions on Industrial Electronics, vol. 58, no. 10, pp. 4857-4865, 2011, doi: 10.1109/TIE.2010.2103538.

[9] Y. S. Can, N. Chalabianloo, D. Ekiz, and C. Ersoy, "Continuous stress detection using wearable sensors in real life: Algorithmic programming contest case study," Sensors, vol. 19, no. 8, p. 1849, 2019.

[10] Y. S. Can, B. Arnrich, and C. Ersoy, "Stress detection in daily life scenarios using smart phones and wearable sensors: A survey," Journal of biomedical informatics, p. 103139, 2019.

[11] A. O. Akmandor and N. K. Jha, "Keep the stress away with SoDA: Stress detection and alleviation system," IEEE Transactions on Multi-Scale Computing Systems, vol. 3, no. 4, pp. 269-282, 2017.

[12] A. Liapis, C. Katsanos, D. Sotiropoulos, M. Xenos, and N. Karousos, "Stress recognition in humancomputer interaction using physiological and selfreported data: a study of gender differences," in Proceedings of the 19th Panhellenic Conference on Informatics, 2015, pp. 323-328.

[13] Akhonda, Md Abu Baker Siddique, S. M. F. Islam, A. S. Khan, F. Ahmed, and M. M. Rahman, "Stress detection of computer user in office like working environment using neural network," in 2014 17th International Conference on Computer and Information Technology (ICCIT), 2014, pp. 174-179.
[14] M. Zubair, C. Yoon, H. Kim, J. Kim, and J. Kim, "Smart wearable band for stress detection," in 2015 5th International Conference on IT Convergence and Security (ICITCS), 2015, pp. 1-4.

[15] Centers for Disease Control and Prevention, Weekly U.S. Influenza Surveillance Report | CDC. [Online]. Available: https://www.cdc.gov/flu/weekly/index.htm (accessed: Jan. 2 2020).

[16] Public Health England, Weekly national flu reports: 2019 to 2020 season. [Online]. Available: https:// www.gov.uk/government/statistics/weekly-nationalflu-reports-2019-to-2020-season (accessed: Jan. 2 2020).

[17] V. Mozgovoy, "Stress Pattern Recognition Through Wearable Biosensors in the Workplace: Experimental Longitudinal Study on the Role of Motion Intensity," in 2019 6th Swiss Conference on Data Science (SDS), 2019, pp. 37-45.

[18] C. M. Bishop, Pattern recognition and machine learning: springer, 2006.

[19] J. Han, J. Pei, and M. Kamber, Data mining: concepts and techniques: Elsevier, 2011.

[20] K. M. Ramachandran and C. P. Tsokos, Mathematical Statistics with Applications: Elsevier Science, 2009.

[21] B. Efron and R. Tibshirani, "Bootstrap Methods for Standard Errors, Confidence Intervals, and Other Measures of Statistical Accuracy," Statist. Sci., vol. 1, no. 1, pp. 54-75, 1986, doi: 10.1214/ss/1177013815.

[22] M. Peligrad, "On the blockwise bootstrap for empirical processes for stationary sequences," Ann. Probab., vol. 26, no. 2, pp. 877-901, 1998, doi: 10.1214/aop/1022855654.

[23] Peter Bühlmann, "Sieve Bootstrap With VariableLength Markov Chains for Stationary Categorical Time Series," Journal of the American Statistical Association, vol. 97, no. 458, pp. 443-471, 2002, doi: 10.1198/016214502760046998.

[24] O. C. Ibe, Fundamentals of applied probability and random processes, 2nd ed. Amsterdam, Boston: Elsevier/AP, 2014.

[25] J. J. Hunter, "Mixing times with applications to perturbed Markov chains," Linear Algebra and its Applications, vol. 417, no. 1, pp. 108-123, 2006.

[26] K. Dery, I. M. Sebastian, and N. van der Meulen, "The Digital Workplace is Key to Digital Innovation," MIS Quarterly Executive, vol. 16, no. 2, 2017.

[27] B. Wojciech, "Digital Era Governance - a new chapter of public management theory and practice," MAZOWSZE Studia Regionalne, no. 22, pp. 117-129, 2017.

[28] V. Mozgovoy and T. Mettler, "Internal development as access strategy to information and communication technology in electronic human resource management for sustaining employee well-being," it-Information Technology, vol. 61, 5-6, pp. 265-272, 2019. 